\title{
Avaliação dos serviços oferecidos pela Estratégia de Saúde da Família (ESF) em um município na região sul do Brasil
}

\author{
Evaluation of the services offered by the Family Health Strategy (ESF) in a municipality in \\ southern Brazil \\ Evaluación de los servicios ofrecidos por la Estrategia Salud de la Familia (ESF) en un municipio \\ del sur de Brasil
}

Recebido: 21/12/2020 | Revisado: 29/12/2020 | Aceito: 02/01/2021 | Publicado: 05/01/2021

Tiago Bittencourt de Oliveira

ORCID: https://orcid.org/0000-0003-4598-0922 Universidade Regional Integrada do Alto Uruguai e das Missões, Brasil E-mail: tiagob@san.uri.br

Vera Regina Medeiros Andrade ORCID: https://orcid.org/0000-0003-4559-8248 Universidade Regional Integrada do Alto Uruguai e das Missões, Brasil E-mail: vandrade@san.uri.br

Fernanda Nascimento Teichmann ORCID: https://orcid.org/0000-0002-3223-5504 Secretaria Municipal de Saúde de Santo Ângelo, Brasil E-mail: fer_teichmann@hotmail.com

Tânia Maria Trevisan Machado ORCID: https://orcid.org/0000-0002-3018-1370 Secretaria Municipal de Saúde de Santo Ângelo, Brasil E-mail: tania.trevisan@ hotmail.com

Andréia Bernardi

ORCID: https://orcid.org/0000-0002-5184-6247 Secretaria Municipal de Saúde de Santo Ângelo, Brasil E-mail: b.deia@hotmail.com

Karen Pietrowski

ORCID: https://orcid.org/0000-0002-4513-0605 Universidade Regional Integrada do Alto Uruguai e das Missões, Brasil E-mail: karen.pietrowski@outlook.com

Larissa Scheeren Thomas

ORCID: https://orcid.org/0000-0002-5890-8105 Universidade Regional Integrada do Alto Uruguai e das Missões, Brasil

E-mail: lari_scheeren_thomas@hotmail.com

Edielli Ricardo Ajala

ORCID: https://orcid.org/0000-0001-7496-2294

Universidade Regional Integrada do Alto Uruguai e das Missões, Brasil E-mail: ajalaedelli2015@hotmail.com

Samuel Felipe Atuati

ORCID: https://orcid.org/0000-0003-0155-098X

Universidade Regional Integrada do Alto Uruguai e das Missões, Brasil

E-mail: atuatis@gmail.com

Francieli Larissa Zamboni

ORCID: https://orcid.org/0000-0002-3671-4051

Universidade Regional Integrada do Alto Uruguai e das Missões, Brasil E-mail: francizamboni@hotmail.com

Tainá Lang Wagner

ORCID: https://orcid.org/0000-0003-2158-1134 Universidade Regional Integrada do Alto Uruguai e das Missões, Brasil E-mail: tainawagner15@gmail.com

Juliane Oliveira Brum

ORCID: https://orcid.org/0000-0003-4928-8335

Universidade Regional Integrada do Alto Uruguai e das Missões, Brasil

E-mail: brumju2000@gmail.com

Andressa Rocha Antunes

ORCID: https://orcid.org/0000-0001-7261-531X Universidade Regional do Alto Uruguai e das Missões, Brasil

E-mail: andressa.antunees@hotmail.com 


\title{
Miriam de Andrade \\ ORCID: https://orcid.org/0000-0001-9934-5444 Universidade Regional do Alto Uruguai e das Missões, Brasil E-mail: milly_deandrade@hotmail.com \\ Narciso Vieira Soares \\ ORCID: https://orcid.org/0000-0002-3860-6943 Universidade Regional Integrada do Alto Uruguai e das Missões, Brasil E-mail: nvsoares@aluno.santoangelo.uri.br \\ Carine Amabile Guimarães \\ ORCID: https://orcid.org/0000-0003-2889-0933 Universidade Regional do Alto Uruguai e das Missões, Brasil E-mail: carine@san.uri.br \\ Izabel Almeida Alves \\ ORCID: https://orcid.org/0000-0002-8935-6542 Universidade Federal da Bahia, Brasil E-mail: izabelalmeidaalves@gmail.com
}

\section{Resumo}

O presente estudo teve como objetivo avaliar os serviços oferecidos em uma Estratégia de Saúde da Família (ESF) do município de Santo Ângelo (RS). Para isto foi realizada uma pesquisa observacional entre outubro de 2019 e março de 2020, pelos integrantes do Programa Educação pelo Trabalho para a Saúde (PET-Saúde-Interprofissionalidade), com 156 participantes. Foram avaliados atributos utilizando o instrumento de Avaliação da Atenção Primária adaptado à realidade local (PCA-Tool adaptado). Dentre os entrevistados os atributos que apresentaram relevância positiva foram grau de afiliação, com $87,6 \%$ dos participantes que procuravam ESF do seu bairro; longitudinalidade, com escore de 8,9; coordenação-sistema de informações, com escore 9,3; integração de cuidados com escore 8,8 e serviços disponíveis na unidade com 8,2; foram os itens mais bem avaliados. Os resultados baixos foram: acessibilidade com escore de 6,9, serviços prestados e orientação comunitária, ambos com escores médios 5,7. O escore geral de avaliação de todos atributos se apresentou em 7,7. Com isto se evidenciou que a ESF apresenta bom desempenho em vários atributos analisados. Porém, com relação à orientação comunitária, integralidade e serviços prestados, apresenta algumas falhas, evidenciando a necessidade de uma mudança de estratégias, focando mais em orientações voltadas à família e comunidade.

Palavras-chave: Sistema único de saúde; Acesso aos serviços de saúde; Integralidade em saúde; Estratégia saúde da família.

\begin{abstract}
This study aimed to evaluate the services offered in a Family Health Strategy (FHS) in the city of Santo Ângelo (RS). For this, an observational research was carried out between October 2019 and March 2020, by the members of the Education through Work for Health Program (PET-Saúde-Interprofessionality), with 156 participants. Attributes were assessed using the Primary Care Assessment instrument adapted to the local reality (adapted PCA-Tool). Among the interviewees, the attributes that showed positive relevance were degree of affiliation, with $87.6 \%$ of the participants looking for FHS in their neighborhood. The longitudinality, with a score of 8.9, coordination-information system with a score of 9.3, integration of care with a score of 8.8 and services available in the unit with 8.2 , were the best rated items. The low results were: accessibility with a score of 6.9 , services provided and community guidance, both with average scores 5.7. The overall score for the evaluation of all attributes was 7.7. It was evidenced that the FHS performs well in several analyzed attributes. However, with regard to community orientation, integrality and services provided, it has some flaws, highlighting the need for a change in strategies, focusing more on guidelines aimed at the family and community.
\end{abstract}

Keywords: Unified health system; Health services accessibility; Integrality in health, Family health strategy.

\section{Resumen}

Este estudio tuvo como objetivo evaluar los servicios ofrecidos en una Estrategia de Salud de la Familia (ESF) en la municipalidad de Santo Ângelo (RS, Brasil). Para ello, se realizó una investigación observacional entre octubre de 2019 y marzo de 2020, por parte de los integrantes del Programa Educación a través del Trabajo para la Salud (PETSaúde-Interprofesionalidad), con 156 participantes. Los atributos se evaluaron utilizando el instrumento de Evaluación de la Atención Primaria adaptado a la realidad local (PCA-Tool adaptado). Entre los entrevistados, los atributos que mostraron relevancia positiva fueron el grado de afiliación, con el 87,6\% de los participantes buscando ESF en su barrio; longitudinalidad, con una puntuación de 8,9; sistema de coordinación-información, con una puntuación de 9,3; integración de la atención con una puntuación de 8,8 y servicios disponibles en la unidad con 8,2; fueron los artículos mejor valorados. Los resultados bajos fueron: accesibilidad con una puntuación de 6,9, servicios prestados y orientación comunitaria, ambos con una puntuación media de 5,7. La puntuación general para la evaluación de todos los atributos fue de 7,7. Con esto se evidenció que la FHS se desempeña bien en varios atributos analizados. Sin embargo, en cuanto a la orientación comunitaria, la integralidad y los servicios prestados, presenta 
algunas fallas, destacando la necesidad de un cambio de estrategias, enfocándose más en orientaciones dirigidas a la familia y la comunidad.

Palabras clave: Sistema único de salud; Accesibilidad a los servicios de salud; Integralidad en salud; Estrategia de salud familiar.

\section{Introdução}

O Sistema Único de Saúde (SUS) está consolidado em seus princípios doutrinários de universalidade, equidade e integralidade (Brasil, 2000). Incorporada ao SUS, a Atenção Primária à Saúde (APS) é a porta de entrada preferencial do usuário para o sistema, minimizando custos, solucionando problemas de competência do primeiro nível de assistência de maneira resolutiva e satisfatória (Matta \& Morosini, 2010; Brasil, 2015). A Estratégia de Saúde da Família (ESF) é uma vantagem para a população, pois proporciona maior universalização, integralização e equidade da assistência, portanto, maior abrangência da APS. Contudo as USFs (Unidades Saúde da Família) apresentam dificuldades, principalmente pelas iniquidades regionais, processos de trabalho baseados no modelo biomédico, e pelo crescimento do setor privado, que impedem que a APS seja realmente a porta de entrada do usuário no setor de saúde (Arantes, Shimizu \& Merchán-Hamann, 2016).

Desse modo, recursos que aprimorem os serviços da APS são de extrema importância para economia do país e para a saúde da população. Então, para que o atendimento seja mais humanizado e de qualidade, é necessário conhecer a satisfação dos usuários dos serviços disponíveis, a fim de que se possam criar estratégias organizativas para aperfeiçoar a qualidade do serviço prestado (Gomes et al., 2016).

A satisfação do usuário com relação ao sistema ou atendimento o define como alguém que pode valorizar peculiaridades importantes para o serviço e a equipe que os atende. Valorizar o relato dado pelo paciente a respeito dos serviços lhe dá um lugar privilegiado como sujeito de direitos e fortalece a cultura participativa (Gomes et al., 2016). Além disso, a humanização do atendimento proporciona segurança e fortifica a relação profissional-paciente, contribuindo inclusive na melhora da saúde do enfermo, oferecendo atendimento de qualidade articulando aos avanços tecnológicos com acolhimento (Hollanda et al., 2012; Brasil, 2004). Em vista disso, a proposta deste estudo corrobora a necessidade de melhorar a escuta e o acolhimento das falas dos indivíduos que compõem este serviço, profissionais e usuários, visando o desenvolvimento de ações na direção de um serviço humanizado, integrado e satisfatório para ambos os lados.

Como instrumento de avaliação da APS no presente estudo utilizamos o Primary Care Assessment Tool (PCATool) que é o instrumento que está sendo mais utilizado no Brasil, pois é uma ferramenta que mais se aproxima da realidade da Estratégia Saúde da Família (ESF). O PCATool foi desenvolvido no centro de pesquisa da Jonhs Hopkins Bloomberg School of Public Health, nos EUA, por Barbara Starfield e colaboradores (Starfield, 2002; Costa, 2020), posteriormente o mesmo foi validado e utilizado para o português. O instrumento identifica se os serviços de saúde e a APS estão orientados segundo seus atributos essenciais e derivados. Os chamados atributos essenciais são elencados como: o acesso de primeiro contato, a longitudinalidade, a integralidade e a coordenação da atenção, estas denominações são assim para aproximação com as ações essenciais da atenção primária em saúde. Já, os atributos derivados são: a orientação familiar, orientação comunitária e competência cultural, que qualificam a APS e ampliam a interação dos indivíduos/comunidade com os serviços de saúde, cabe dizer que a competência cultural não faz parte da versão em português, pois não encontrou representação nos estudos de validação (Araújo-Filho et al., 2019)

Diante do exposto, o objetivo desse estudo foi avaliar os serviços oferecidos por uma USF (Unidade Saúde da Família) do município de Santo Ângelo (RS). 


\section{Metodologia}

Trata-se de uma pesquisa transversal, exploratória e descritiva com abordagem quantitativa, realizada no período de outubro de 2019 a março de 2020, na USF do bairro São Carlos, município de Santo Ângelo, Rio Grande do Sul, Brasil. A pesquisa foi realizada pelo Grupo Gestão em Saúde e Educação Permanente com Docentes e Trabalhadores do SUS, no Programa de Educação pelo Trabalho para a Saúde PET-Saúde-Interprofissionalidade, com Cursos da Área da Saúde, Educação Física, Enfermagem, Farmácia e Psicologia da Universidade Regional Integrada do Alto Uruguai e das Missões, Campus de Santo Ângelo.

A população foi formada pelo número total de usuários residentes na área geográfica coberta pela ESF, e a amostra ( $\mathrm{n}=156$ participantes), calculada com base na população de usuários $(\mathrm{n}=2.923)$ cadastrados na USF. Para isso, utilizou-se fórmula para populações finitas, estimando frequência hipotética do fator do desfecho na população (proporção de alto escore de APS entre as unidades, $\mathrm{p}=50 \pm 5$ ), com intervalo de confiança de $80 \%$, margem de erro de $5 \%$, com a ferramenta on-line gratuita OpenEpi® (Sullivan, Dean \& Soe, 2009).

Foram incluídos participantes que residiam na área adscrita da ESF, utilizavam algum serviço da unidade, com idade $\geq 18$ anos, e excluídos os que não se prontificaram a responder ao questionário. $\mathrm{O}$ recrutamento dos participantes foi por conveniência, de forma não-probabilística. Foram feitos convites aos usuários presentes na USF e busca ativa por meio de visitas domiciliares. Os participantes que aceitavam participar da pesquisa assinavam o Termo de Consentimento Livre e Esclarecido (TCLE) e, após, respondiam ao questionário. O projeto de pesquisa foi aprovado pelo Comitê de Ética da Universidade Regional Integrada do Alto Uruguai e das Missões (URI), Santo Ângelo, conforme parecer consubstanciado número 3.573.991.

Foi utilizado, para a coleta dos dados, o Instrumento de Avaliação da Atenção Primária (Primary Care Assessment Tool - PCATool), validado e publicado no Manual do Instrumento de Avaliação da Atenção Primária à Saúde, do Ministério da Saúde (Brasil, 2010). Esse instrumento aborda elementos essenciais (acesso de primeiro contato, longitudinalidade, integralidade e coordenação da atenção) e alguns derivativos (orientação familiar, orientação comunitária e competência cultural) (Starfield, 2002). O questionário utilizado na pesquisa foi adaptado para a realidade local e contou com 29 questões, nos 10 eixos elencados pelo instrumento.

Cada atributo essencial é constituído por dois componentes ou subdimensões, um relacionado à estrutura e outro ao processo. Os itens apresentam respostas em escala Likert, de 4 pontos (4 para "com certeza sim"; 3 para "provavelmente, sim"; 2 para "provavelmente, não"; 1 para "com certeza não" e 9 para "não sei/não lembro"), resultando em escores para cada atributo. O escore final de cada um dos atributos é realizado pela média das respostas de seus itens (Costa, 2020).

O questionário utilizado na pesquisa foi adaptado pelos autores, para realidade local, e contou com 29 questões, nos 10 eixos elencados pelo instrumento, constituiu de 1 questão em cada eixo A e B, 3 questões em cada eixo C, D e E, 1 questão no eixo F, 9 questões no eixo G, 4 questões no eixo H, 1 questão no eixo I e 3 questões no eixo J. Para os itens do eixo C, a questão C9, foi formulada de maneira que quanto maior o valor (resposta) atribuído, menor é a orientação para APS, logo, este item teve seu valor invertido para: (valor $4=1$ ), (valor $3=2$ ), (valor $2=3$ ) e (valor $1=4$ ).

Se para um entrevistado, a soma de respostas em branco ("missing") com respostas "9" ("não sei/não lembro") atingir $50 \%$ ou mais do total de itens de um componente, não é calculado o escore deste componente para este entrevistado, ficando em branco ("missing") no banco de dados. Se para um entrevistado, a soma de respostas em branco ("missing") com respostas “9" ("não sei/não lembro") for inferior a 50\% do total de itens de um componente, transforma-se o valor "9" para valor "2" ("provavelmente não"). Esta transformação é necessária para pontuar negativamente algumas características do serviço de saúde que não são conhecidas pelo entrevistado (Brasil, 2010). 
Os dados foram organizados em um banco de dados e analisados por meio do programa Statistical Package for the Social Sciences® (SPSS), versão 17.0; foram analisados os dados absolutos e percentuais e calculadas as médias, desvios padrões dos escores de domínios e geral.

Os escores para cada atributo ou seus componentes foram calculados conforme o indicado pelo PCATool, respeitando as adaptações realizadas no questionário. Foram calculados os escores pela média aritmética simples dos valores das respostas dos itens que compõem cada atributo ou seu componente. Para transformar os escores em escala de 0 a 10 foi utilizada a seguinte fórmula: [escore obtido-1(valor mínimo)]×10/4(valor máximo-1(valor mínimo).

Os escores foram obtidos a partir das perguntas dos domínios e variaram de 0 a 10 . Foram considerados valores inferiores a 6,6 como baixo escore e iguais ou superiores a este número como alto (Castro et al., 2012; Chomatas et al., 2013; Oliveira, 2012). O escore geral foi calculado a partir da média dos componentes (Costa, 2020).

\section{Resultados}

Com relação ao grau de afiliação, dos 153 participantes, 87,6\% (n=134) geralmente procuram a USF quando ficam doentes ou precisam de conselhos sobre saúde, seguido do HSA (7,2\%).

Tabela 1 - Distribuição do Grau de Afiliação dos usuários da ESF São Carlos, Santo Ângelo (RS), Brasil (n=153).

Atributo A - Grau de afiliação

\begin{tabular}{lcc}
\hline $\begin{array}{l}\text { A1 - Onde você geralmente vai quando fica doente ou precisa de conselhos sobre a } \\
\text { sua saúde? }\end{array}$ & $\mathrm{n}$ & $\%$ \\
\hline USF São Carlos & 134 & 87,6 \\
Hospitais (HSA e H-UNIMED) & 11 & 7,2 \\
UPA & 4 & 2,6 \\
Outros & 4 & 2,6 \\
\hline Total & 153 & 100 \\
\hline
\end{tabular}

Fonte: Autores.

Na Tabela 2 estão apresentadas as frequências das respostas dos usuários a cada item dos atributos medidos pelo PCATool, com seus respectivos escores médios (Figura 1). 
Figura 1 - Descrição dos valores dos escores obtidos em cada atributo.

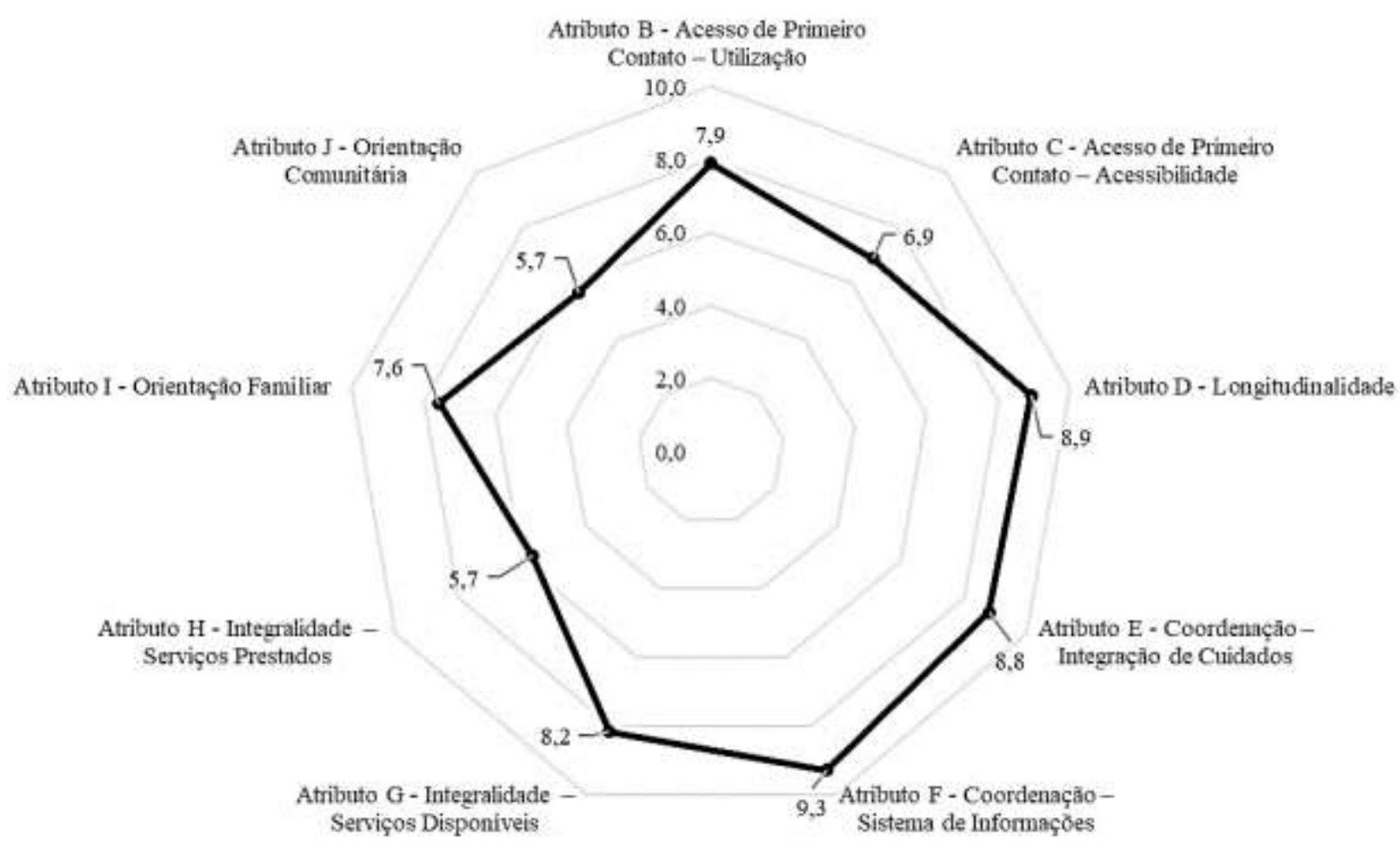

Fonte: Dados da pesquisa. 
Tabela 2 - Distribuição das frequências das respostas dos usuários da ESF São Carlos, Santo Ângelo (RS), Brasil, a cada item dos atributos medidos pelo PCATool, com seus respectivos escores médios.

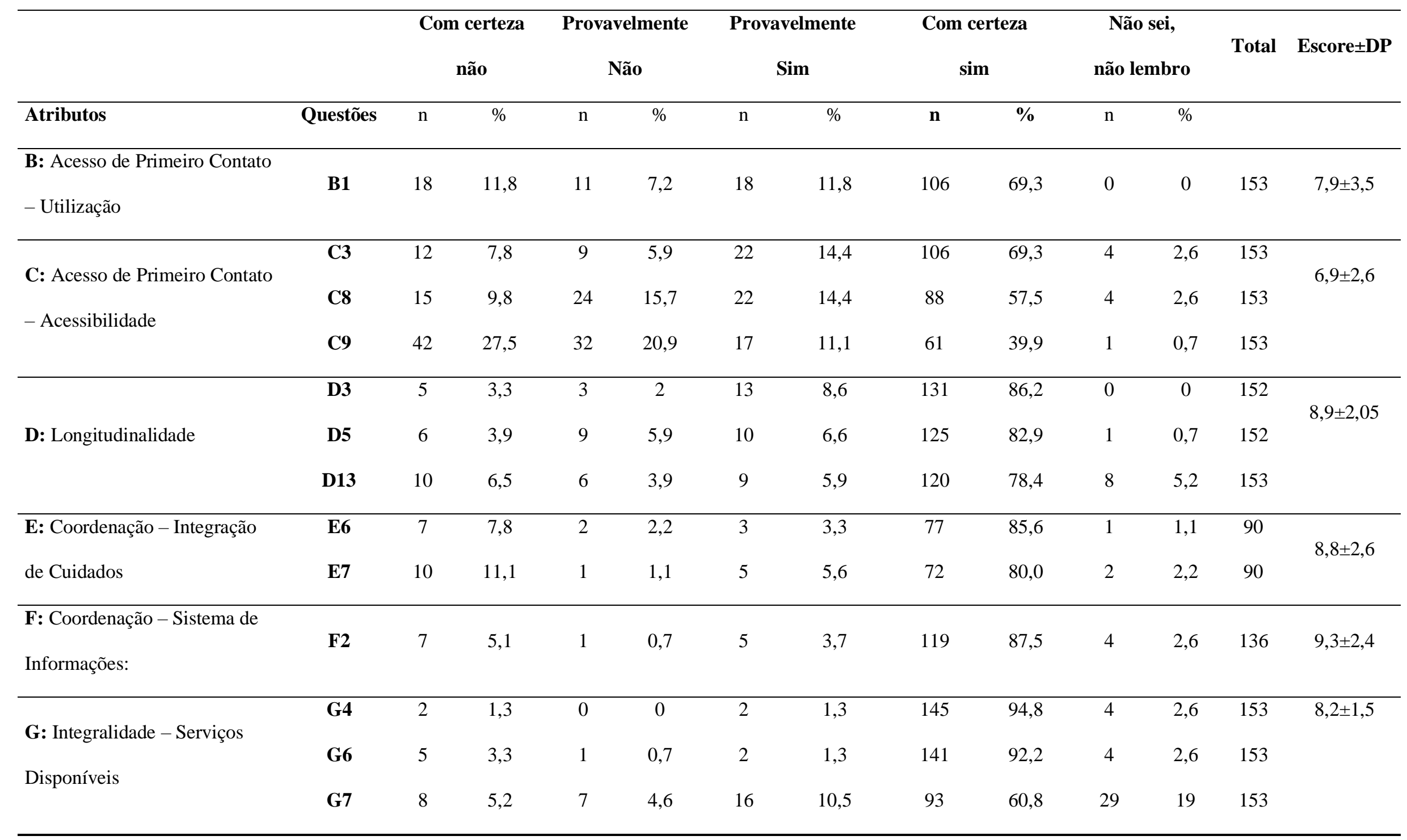


Research, Society and Development, v. 10, n. 1, e13310111409, 2021

(CC BY 4.0) | ISSN 2525-3409 | DOI: http://dx.doi.org/10.33448/rsd-v10i1.11409

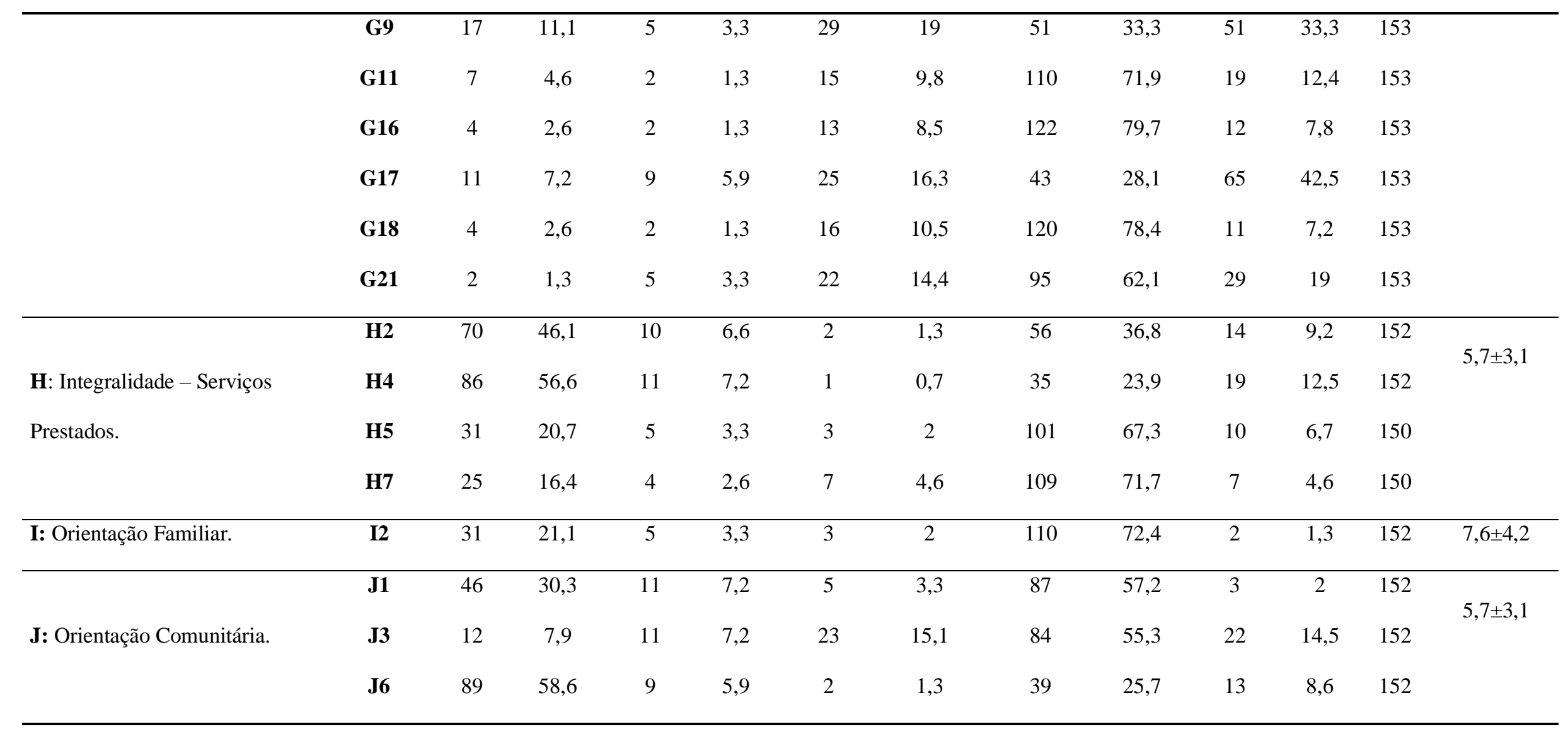

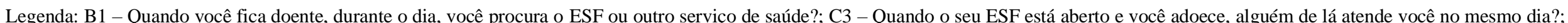
C8 - É fácil marcar hora para uma consulta de revisão neste ESF?; C9 - Quando você chega no seu ESF, você tem que esperar mais de 30 minutos para se consultar com o médico ou enfermeiro, após o acolhimento?; D3 - O seu médico/enfermeiro responde suas perguntas de maneira que você entenda?; D5 - Durante a consulta, você tem tempo suficiente para falar sobre seus problemas de saúde?, D13 - O seu médico/enfermeiro sabe a respeito de todos os medicamentos que você está tomando?; E6 - Quando você precisou ser encaminhado para serviço especializado, o seu médico/enfermeiro escreveu alguma informação, a respeito do motivo deste encaminhamento?, E7 - O seu médico/enfermeiro sabe quais foram os resultados da consulta com o especialista?; F2. Quando você vai consultar, o seu prontuário (história clínica) está sempre disponível na consulta?; G4 - Vacinas (imunizações); G6 - Tratamento dentário; G7 - Planejamento familiar ou métodos anticoncepcionais; G9 - Aconselhamento para problemas de saúde mental; G11 - Aconselhamento e solicitação de teste rápidos (HIV, Sífilis, HB, HC); G16 - Exame preventivo para câncer de colo de útero (teste Papanicolau), G17; Aconselhamento sobre como parar de fumar; G18 - Cuidados pré-natais; G21 - Orientações sobre cuidados no domicílio para alguém da sua família como: curativos, troca de sondas, banho na cama; H2 - Segurança no lar, como guardar medicamentos, substâncias perigosas (ex.: veneno para formiga/rato, água sanitária), arma de fogo e prevenir queimaduras?; H4 - Maneiras de lidar com conflitos de família; H5 - Conselhos a respeito de exercícios físicos apropriados para você; H7 - Verificar e discutir os medicamentos que você está tomando; I2 - O seu médico/enfermeiro já lhe perguntou a respeito de doenças na família (câncer, alcoolismo, depressão)?; J1 - Alguém no ESF faz visitas domiciliares?; J3 - O seu ESF ouve

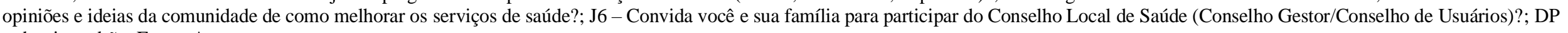
- desvio padrão. Fonte: Autores. 


\section{Discussão}

Com relação ao Grau de Afiliação, a maioria dos entrevistados (87,6\%) no presente estudo respondeu que procura sua unidade como serviço de saúde primário, esses resultados são similares ao estudo de Vidal et al. (2012), em que 68,4\% dos entrevistados utilizavam os Centros de Saúde como serviço de referência, 9,7\% a UPA e 2,2\% a policlínica municipal. É recomendado que a prioridade seja a Atenção Primária à Saúde (APS), pois utiliza a humanização na relação equipe e população e, por meio da avaliação de risco e vulnerabilidade, esse tipo de atendimento deve se tornar o primeiro elo da rede assistencial (Vidal et al., 2012). A antecipação de procura por hospitais para atendimento primário sobrecarrega o sistema, gera transtornos desnecessários aos usuários e uso irracional dos recursos disponíveis (Faria, 2014).

Os resultados citados anteriormente concordam com os obtidos no atributo Acesso de Primeiro Contato - Utilização, que demonstraram que a maioria $(69,3 \%)$ dos participantes procura sua unidade como primeiro contato, apresentando escore de 7,9 $( \pm 3,5)$. Este dado é próximo ao encontrado por Motizuki (2016) que apresentou um escore de 9,5. Ressaltando-se dessa forma a importância da ESF como porta de entrada para a rede assistencial de saúde, desempenhando funções com base nos princípios que regem o SUS.

Ao serem questionados sobre o atributo Acesso de Primeiro Contato - Acessibilidade, "alguém de lá atende você no mesmo dia?" 69,3\% dos participantes responderam "com certeza sim"; com relação à facilidade para marcar consultas de revisão, 57,5\% responderam "com certeza sim", e sobre o tempo de espera para realização da consulta, 39,9\% afirmam ter que esperar mais de 30 minutos, com escore de 6,9 $( \pm 2,6)$. Alguns dados não estão conforme o estudo de Sala et al. (2011), no qual $62 \%$ relataram ser muito difícil marcar uma consulta não emergencial em menos de 24 horas e $68 \%$ dos usuários disseram esperar sempre mais de 30 minutos. Já para a facilidade ao marcar consultas de revisão, está conforme, pois $72 \%$ dos entrevistados relataram sempre conseguir marcar este tipo de consulta.

Na pesquisa realizada por Pereira et al. (2011), com relação ao tempo de espera, 20,4\% dos entrevistados relataram ter que esperar mais de 30 minutos para consulta. Nossos dados discordam do estudo de Costa (2020), em que 46,4\% ( $\mathrm{n}=108$ ) conseguem ser atendidos no mesmo dia, em caso emergencial, com escore de 2,8. Essa demora no atendimento traz consequências negativas tanto para o indivíduo quanto para a população. Levando em consideração o indivíduo, grande parte dos usuários precisa faltar o trabalho para ir à consulta devido à demora no atendimento, prejudicando suas atividades ocupacionais. Já as consequências a nível de população são maiores (Reis et al., 2013).

Com relação ao atributo longitudinalidade, $86,2 \%$ dos participantes responderam que médicos e enfermeiros respondem de maneira compreensível. Além disso, 82,9\% dos entrevistados declaram que o tempo é suficiente para as consultas, o escore deste atributo foi de 8,91 $( \pm 2,05)$. Kessler et al. (2018), no estudo para avaliar a longitudinalidade do cuidado na APS na perspectiva dos usuários, apresentou um escore de 6,9, considerado alto. Na pesquisa de Costa (2020), o escore médio obtido no atributo Longitudinalidade foi de 6,4. Enquanto Souza et al. (2019), no estudo sobre o desempenho da ESF com usuários cadastrados, obtiveram um escore de 5,8, considerado baixo. O resultado do presente estudo demonstra a satisfação dos pacientes com o atendimento médico recebido. Em virtude disso, os pacientes se beneficiam com um bom atendimento, e a USF se beneficia com o retorno de seus pacientes pela qualidade dos serviços prestados.

Quanto maior a atenção prestada pelos médicos durante a consulta, maior a satisfação dos pacientes em relação ao serviço; além do sentimento de valorização quando são ouvidos atentamente. Um dos motivos deste alto escore do nosso estudo pode ser explicado pelo fato de que os usuários entrevistados reconhecem os profissionais como suporte de apoio, em virtude de que se preocupam em prestar assistência adequada. Além disso, o tamanho da população assistida pela USF pode influenciar na discrepância entre os estudos. Kessler et al. (2019) afirma que os resultados menos otimistas são encontrados nos municípios de maior porte, e isso ocorre pelo fato de que grandes municípios apresentam menores coberturas de ESF, superlotação e, consequentemente, redução da atenção no atendimento. 
O atributo Coordenação - Integração de Cuidados apresentou neste estudo escore de 8,8 (ะ2,6), 59,6\% dos usuários já foram encaminhados para um serviço especializado de saúde, 85,6\% relataram que o médico/enfermeiro escreveu informações sobre o motivo do encaminhamento. Os dados estão conforme o estudo realizado por Silva (2017), Silva et al. (2018) e Costa (2020), que obtiveram resultados muito semelhantes. Em relação ao escore, é semelhante ao encontrado por Costa (2020) de 7 $( \pm 2,8)$, classificado como alto. Os percentuais positivos elevados demonstram que os usuários estavam satisfeitos com os encaminhamentos aos especialistas, e que a Rede de Atenção à Saúde auxilia na resolubilidade dos casos. O encaminhamento às especialidades e aos hospitais deve ser realizado pela unidade básica de saúde em decorrência da triagem clínica do paciente, considerando sempre a gravidade do problema, para que dessa forma possa ser analisado pelas centrais de regulação (Brasil 2009).

Nossos achados do atributo Coordenação - Sistemas de Informações não concordam com os valores encontrados por Costa (2020), em que 12,4\% dos usuários entrevistados responderam que poderiam ler ou consultar o seu prontuário caso quisessem. O nosso escore de 9,3 $( \pm 2,4)$ também difere do que Costa $(2020)$ registrou: 5,9 $( \pm 2,5)$. Essa divergência encontrada pode ser devido à não informatização completa dos processos referentes à APS e à passagem dos usuários em outros serviços de saúde no estado. Ainda que os prontuários eletrônicos sejam utilizados para armazenar informações sobre os laudos dos exames, em alguns locais o prontuário é impresso (Gonçalves et al., 2013). Os sistemas de informações presentes no SUS envolvem vários processos, como; capturar, processar, transformar, armazenar, manter e produzir informações. Além disso, é relevante para a obtenção de informações necessárias, de forma que os serviços de saúde consigam desenvolver e aplicar estratégias que auxiliam em condições melhores para planejamento, e avaliação de ações que corroboram para melhorias na área da saúde (Silva et al., 2018). Dessa forma, o sistema eletrônico garante funcionalidade quando pensamos em grandes cidades que necessitam de gerenciamento em larga escala (Gonçalves et al., 2013).

No atributo Integralidade - Serviços disponíveis, entre 60\% e 95\% responderam "com certeza sim" quando questionados sobre disponibilidade dos serviços disponíveis. Porém, sobre aconselhamento para problemas de saúde mental e aconselhamento sobre tabaco, os percentuais de respostas "não sei/não lembro" foram de 33,3\% e 42,5\%, respectivamente. Evidenciou-se o elevado escore com relação a estes serviços $8,2( \pm 1,5)$, que se apresenta bem satisfatório; contudo esse resultado acabou sendo diferente de outros estudos que utilizaram o mesmo instrumento para avaliação. Um estudo que avaliou a qualidade dos serviços de atenção básica, na perspectiva de usuários adultos, identificou um escore de 5,3 com relação aos serviços disponíveis (Costa, 2020), além disso, uma revisão integrativa da literatura sobre a avaliação da qualidade dos serviços da APS analisou 17 artigos e obteve uma média de 4,8 sobre os serviços disponíveis (Araujo Filho et al., 2019). Esses estudos abordaram a indisponibilidade de políticas e serviços com estratégias específicas; os usuários acabam procurando os serviços para tratamentos mais especializados, que necessitam de mais recursos tecnológicos (Costa, 2020; Araujo Filho et al., 2019), demonstrando que no presente estudo há maior disponibilidade e conscientização dos serviços e dos profissionais para as atividades abordadas, bem como maior procura dos usuários para esses atendimentos.

De acordo com os resultados obtidos em Integralidade - Serviços Prestados, na questão a respeito de "Segurança do lar, como guardar medicamentos, substâncias perigosas, armas de fogo e como prevenir queimaduras”, constatou-se a não prestação ou não conhecimento dos serviços abordados por boa parte das famílias, visto que 46,1\% responderam "com certeza, não" para a prestação do serviço. É claramente um aspecto preocupante, tendo em vista o número crescente de óbitos por intoxicação e hospitalizações por essa causa, assim como suicídios. Nascimento, Pimentel e Adaid-Castro (2019) apontaram para um total de 332.795 homicídios por arma de fogo entre 1999 e 2008, com muitos destes casos tendo ocorrido em ambiente familiar. Destaque também às queimaduras, resultados da ação de diversos efeitos do cotidiano, em especial para as crianças. Mais da metade $(56,6 \%)$ dos entrevistados responderam não ter orientações sobre como lidar com conflitos familiares, demonstrando de forma insatisfatória a prestação desse serviço. A maioria dos entrevistados $(67,3 \%)$ respondeu que recebe 
aconselhamento sobre exercícios físicos. Sabe-se que a urbanização e comodidade da população agrava os quadros de sedentarismo, implicando doenças como hipertensão arterial, obesidade e diabetes (Santos et al., 2012). A educação em saúde e a prática de exercícios físicos apresentam um potencial de mudança acompanhado por demais fatores, como melhora no estilo de vida e consequentemente prevenção de tais doenças (Santos et al., 2012). Sobre os medicamentos, 71,7\% dos entrevistados confirmaram que existe verificação e discussão sobre os medicamentos que utilizam. Este é um fator determinante para a diminuição de taxas de óbitos e hospitalizações decorrentes da automedicação e uso irracional de medicamentos.

O escore de Serviços Prestados correspondeu a 5,7, revelando-se baixo e de acordo com a análise de Araujo Filho et al. (2019); com média de 6,2, destacaram, no quadro descrito, a negligência ou insatisfação. A falta da prestação de alguns serviços pode ocasionar descuidos ou negligências, prejudicando a população. Desta forma, faz-se necessário capacitar as equipes de saúde pública sobre cuidados na segurança do lar com as famílias assistidas (Souza et al., 2019). A inserção de cartazes explicativos e de preferência ilustrados, em locais de acesso do paciente nas unidades básicas de saúde, abordando estes temas, poderia resultar na diminuição dessas causalidades (Motizuki, 2016). São assuntos que devem ser tratados com seriedade e acompanhamento profissional, e se esta fragilidade é reconhecida por gestores e profissionais de saúde, podem ser desenvolvidas novas estratégias e práticas de cuidado, além de promover maior discussão sobre o tema (Kesler et al., 2018).

No atributo Orientação Familiar, 72,4\% dos entrevistados responderam que são questionados a respeito de doenças na família. Esse questionamento faz parte da anamnese que deve ser realizada durante o atendimento ao paciente (Santos, Veiga \& Andrade, 2011). A média do escore encontrado para a orientação familiar foi de 7,6 ( $\pm 4,2)$, discordando do estudo realizado por Costa (2020), que obteve um escore médio de $4,9( \pm 3,1)$, em que a maioria dos entrevistados $(62,2 \%, n=145)$ respondeu que o profissional de saúde não orienta sobre cuidar de si ou de um familiar. Neste atributo, nosso escore médio é extremamente satisfatório, pois demonstra o bom funcionamento do atendimento.

Para o atributo Orientação Comunitária, 57,2\% dos participantes do presente estudo responderam que recebiam visitas domiciliares; 55,3\% responderam que os profissionais ouvem suas opiniões e ideias sobre os serviços de saúde; porém 58,6\% nunca foram convidados a participar do Conselho Local de Saúde, e o escore médio foi de 5,7 $( \pm 3,1)$, considerado baixo. Segundo Reichert et al. (2016), as necessidades relacionadas à saúde das pessoas ocorrem em um contexto social, e a percepção dessas necessidades requer o conhecimento desta realidade; diferente do apresentado por Alencar et al. (2014), que avaliaram a satisfação por parte dos usuários sobre as visitas domiciliares. A visita domiciliar está ligada a essa tomada de opiniões, pois é um instrumento usado para que os serviços consigam se aproximar e identificar as necessidades das famílias dentro da comunidade, auxiliando nas medidas que devem ser tomadas (Daschevi et al., 2015). Isso mostra que as porcentagens próximas obtidas quando questionados sobre visitas domiciliares e tomada de opiniões estão interligadas. A participação da comunidade nos conselhos de saúde é restrita a parte dos usuários da $\mathrm{AB}$, pois muitos podem desconhecer sobre a participação comunitária nesse tipo de organização pela não divulgação dessa possibilidade (Daschevi et al., 2015; Oliveira, 2007) corroborando nossos achados. Um escore baixo evidencia que os princípios básicos de uma ESF com foco na família e comunidade não estão sendo seguidos em plenitude, podendo refletir na perpetuação de uma atenção pautada no modelo individual e curativo (Oliva et al., 2015). Desta forma, a ESF necessita aumentar a comunicação com seus usuários, convidando-os a participar do conselho de saúde municipal.

O escore geral da presente pesquisa foi de 7,9, superior ao de outros estudos (Costa, 2020; Vidal et al., 2018; Souza et al., 2019; Araujo Filho et al., 2019). O nível de qualidade apresentado no escore geral talvez reflita as condições de assistência da população. A ESF em questão assiste 2.800 pessoas, situação melhor que o preconizado pela Política Nacional de Atenção Básica, a qual define que cada equipe de saúde da família deve ser responsável por, no máximo, 4 mil pessoas, sendo a média recomendada de 3 mil (Brasil, 2007). 


\section{Conclusão}

Baseado nos resultados apresentados, evidenciou-se que a USF apresenta bom desempenho no escore geral, o que em vários atributos analisados foram bem avaliados como o acesso ao serviço, longitudinalidade (cuidado e orientações da equipe de saúde), integração do cuidado (acesso a especialidades médicas e retorno ao USF), sistema de informação e de serviços disponíveis (aplicação de vacinas, tratamento dentário, testes rápidos, cuidados pré-natais, exame preventivo de Papanicolau, entre outros).

Porém, com relação aos atributos de orientação comunitária, integralidade e serviços prestados, apresenta algumas falhas, com baixos índices destacados a falta de orientação quanto as maneiras de lidar com conflitos de família, falta de orientação quanto a segurança no lar - como guardar medicamentos, substâncias perigosas (ex.: veneno para formiga/rato), arma de fogo e prevenção de queimaduras. Além disso, a USF deve apresentar melhoria no aconselhamento da saúde mental e, não há, orientação quanto a participação dos usuários no Conselho local de Saúde. Isto tudo evidencia a necessidade de uma mudança de estratégias, focando mais nestas orientações voltadas à família e a comunidade.

\section{Agradecimentos}

Ao Ministério da Saúde, Secretaria de Gestão do Trabalho e da Educação na Saúde (SGTES). Programa de Educação pelo Trabalho para a Saúde PET-Saúde/Interprofissionalidade - 2018/2020.

\section{Referências}

Alencar, M. N., Coimbra, L. C., Morais, A. P. P., Silva, A. A. M., Pinheiro, S. R. A., \& Queiroz, R. C. S. (2014). Evaluation of the family focus and community orientation in the Family Health Strategy. Ciênc Saúde Coletiva, 19 (2), 353-64. http://dx.doi.org/10.1590/1413-81232014192.08522012.

Arantes L. J., Shimizu H. E., \& Merchán-Hamann, E. (2016). Contribuições e desafios da Estratégia Saúde da Família na Atenção Primária à Saúde no Brasil: revisão da literatura. Ciênc Saúde Coletiva, 21 (5), 1499-510. http://dx.doi.org/10.1590/1413-81232015215.19602015.

Araujo Filho, A. C. A., Nadabe e Silva, A., Ribeiro, M. G. C., Rocha, S. S., Andrade, A. M. L. R., \& Nogueira, L. T. (2019). Avaliação da Atenção Primária à Saúde sob a ótica de cuidadores de crianças: revisão integrativa. Rev esc enferm USP, 1 (53), 27-35. http://dx.doi.org/10.1590/s1980-220x2018030003527

Castro, R. C. L., Knauth, D. R., Harzheim, E., Hauser, L., \& Duncan, B. B. (2012). Avaliação da qualidade da atenção primária pelos profissionais de saúde: comparação entre diferentes tipos de serviços. Cad Saúde Pública, 28 (9), 1772-84. http://dx.doi.org/10.1590/S0102-311X2012000900015

Chomatas, E., Vigo, A., Marty, I., Hauser, L., \& Harzheim, E. (2013). Avaliação da presença e extensão dos atributos da atenção primária em Curitiba. Rev Bras Med Fam Comunidade, 8 (29), 294-303. https://doi.org/10.5712/rbmfc8(29)828

Comes, Y., Trindade, J. S., Shimizu, H. E., Hamann, E. M., Bargioni, F., Ramirez, L., et al. (2016). Avaliação da satisfação dos usuários e da responsividade dos serviços em municípios inscritos no Programa Mais Médicos. Ciênc Saúde Coletiva, 21 (9), 2749-59. http://dx.doi.org/10.1590/141381232015219.16202016

Conselho Nacional de Secretários de Saúde (2015). A Atenção Primária e as redes de atenção à saúde [Internet]. Brasília: CONASS [citado 2020 jul 15]. https://www.conass.org.br/biblioteca/pdf/A-Atencao-Primaria-e-as-Redes-de-Atencao-a-Saude.pdf

Costa L. B. (2020). Avaliação da qualidade dos serviços públicos de atenção primária à saúde em Fortaleza-CE, 2020 [dissertação]. Fortaleza (CE): Universidade Federal do Ceará. $\quad$ http://repositorio.ufc.br/handle/riufc/50882\#: :text=B.,Avalia\%C3\%A7\%C3\%A3o\%20da\%20qualidade\%20dos\%20servi\%C3\%A7os\%20p\%C3\%BAblicos\%20de\%20aten\%C3\%A7\%C3\%A3o\%20prim\%C3\%A1 ria\%20\%C3\%A0,2020.\&text=O\%20PCATool\%20(Primary\%20Care\%20Assessment,partir\%20da\%20perspectiva\%20do\%20usu\%C3\%A1rio.

Daschevi, J. M., Tacla, M. T. G. M., Alves, B. A., Toso, B. R. G. O., \& Collet, N. (2015). Avaliação dos princípios da orientação familiar e comunitária da atenção primária à saúde da criança. Semina: Ciênc Biol Saúde, 36 (1), 31-8. http://dx.doi.org/10.5433/1679-0367.2015v36n1p31

Faria, R. M. (2014). A atenção primária, o território e as redes de atenção: intercambiamentos necessários para a integração das ações do sistema único de saúde (SUS) Em Minas Gerais, Brasil. Hygeia, 10 (19), 8-23. http://www.seer.ufu.br/index.php/hygeia/article/view/26282.

Gonçalves, J. P. P., Batista, L. R., Carvalho, L. M., Oliveira, M. P., Moreira, K. S., \& Leite, M. T. S. (2013). Prontuário Eletrônico: uma ferramenta que pode contribuir para a integração das Redes de Atenção à Saúde. Saúde Debate, 37 (96), 43-50. http://dx.doi.org/10.1590/S0103-11042013000100006

Hollanda, E., Siqueira, S. A. V., Andrade G. R. B., Molinaro, A., \& Vaitsman, J. (2012). Satisfação e responsividade em serviços de atenção à saúde da Fundação Oswaldo Cruz. Ciênc Saúde Coletiva, 17 (12), 3343-52.

Instituto Brasileiro de Geografia e Estatística (2017). Pesquisa: habitantes municípios e estabelecimentos do SUS. Rio de Janeiro: IBGE. https://cidades.ibge.gov.br/ 
Kessler, M., Lima, S. B. S., Weiller, T. H., Lopes, L. F. D., Ferraz, L., Eberhardt, T. D., et al. (2019). Longitudinalidade do cuidado na atenção primária: avaliação na perspectiva dos usuários. Acta Paul Enferm, 32 (2), 186-93. https://doi.org/10.1590/1982-0194201900026

Kessler, M., Lima, S. B. S., Weiller, T. H., Lopes, L. P. D., Ferraz, L., \& Thumé, E. (2018). A longitudinalidade na Atenção Primária à Saúde: comparação entre modelos assistenciais. Rev Bras Enferm. 71 (3), 1063-71. https://doi.org/10.1590/0034-7167-2017-0014

Matta, G. C., \& Morosini, M. V. G. (2010). Atenção primária à saúde. In: Pereira, I. B., Lima, J. C. F. organizadores. Dicionário da educação profissional em saúde. Rio de Janeiro: EPSJV, 23-8. https://www.arca.fiocruz.br/handle/icict/25955

Ministério da Saúde (2009). Portaria $\mathrm{n}^{\circ} 1.820$, de 13 de agosto de 2009. Dispõe sobre os direitos e deveres dos usuários da saúde [Internet]. Diário Oficial da União, Brasília (DF), 13 ago 2009 [citado 2020 jul 13]. http://bvsms.saude.gov.br/bvs/saudelegis/gm/2009/prt1820_13_08_2009.html

Ministério da Saúde. Departamento de Atenção Básica (2010). Manual do instrumento de avaliação da atenção primária à saúde: primary care assessment tool, PCATool-Brasil. Brasília: Ministério da Saúde. http://189.28.128.100/dab/docs/portaldab/documentos/20200506_Pcatool_versao_preliminar_Final.pdf

Ministério da Saúde. Secretaria de Atenção à Saúde. Departamento de Atenção Básica (2007). Política Nacional de Atenção Básica [Internet]. (4a ed.) Brasília: Ministério da Saúde; 2007 [citado 2020 jul 15]. http://bvsms.saude.gov.br/bvs/publicacoes/politica_nacional_atencao_basica_4ed.pdf

Ministério da Saúde. Secretaria Executiva (2000). Sistema Único de Saúde (SUS): princípios e conquistas [Internet]. Brasília: Ministério da Saúde [citado 2020 jul 15]. http://bvsms.saude.gov.br/bvs/publicacoes/sus_principios.pdf

Ministério da Saúde. Secretaria-Executiva. Núcleo Técnico da Política Nacional de Humanização (2004). Humaniza SUS: Política Nacional de Humanização: a humanização como eixo norteador das práticas de atenção e gestão em todas as instâncias do SUS [Internet]. Brasília: Ministério da Saúde [citado 2020 jul 15]. http://bvsms.saude.gov.br/bvs/publicacoes/humanizasus_2004.pdf

Motizuki, L. A. C. (2016). Análise dos atributos da Atenção Primária através do Primary Care Assessment Tool (PCATool- Brasil) [dissertação]. Campo Grande (MS): Universidade Federal de Mato Grosso do Sul. http://www.index-f.com/lascasas/documentos/lc0799.pdf

Nascimento, T. G., Pimentel, C. E., \& Adaid-Castro, B. G. (2016). Escala de Atitudes frente à Arma de Fogo (EAFAF): Evidências de Sua Adequação Psicométrica. Psic: Teor Pesqui, 1 (32), 239-348. https://www.scielo.br/scielo.php?pid=S0102-37722016000100239\&script=sci_abstract\&tlng=pt

Oliva, A. C. D., Moura, C. M. R., Lima, C. A., Costa, F. M., \& Rocha, J. F. D. (2015). Avaliação dos atributos do cuidado primário de saúde na perspectiva do usuário. Rev Uniabeu, 8 (18), 196-208. https://revista.uniabeu.edu.br/index.php/RU/article/view/1750/pdf_191

Oliveira, M. M. C. (2007). Presença e extensão dos atributos da atenção primária à saúde entre os serviços de atenção primária em Porto Alegre: uma análise agregada [dissertação]. Porto Alegre (RS): Universidade Federal do Rio Grande do Sul. https://lume.ufrgs.br/handle/10183/12649

Oliveira, V. B. C. A. (2013). Avaliação da atenção primária à saúde da criança no município de Colombo - Paraná, 2012 [dissertação]. São Paulo (SP): Universidade de São Paulo. https://teses.usp.br/teses/disponiveis/7/7141/tde-22022013-125217/pt-br.php

Pereira, M. J. B., Abrahão-Curvo, P., Fortuna, C. M., Coutinho, S. S., Queluz, M. C., Campos, L. V. O., et al. (2011). Avaliação das características organizacionais e de desempenho de uma unidade de atenção básica à saúde. Rev Gaúcha Enferm., 32 (1), 48. https://doi.org/10.1590/S198314472011000100006

Reichert, A. P. S., Leônico, A. B. A., Toso, B. R. G., Santos, N. C. C. B., Vaz, E. M. C., Collet, N. (2016). Orientação familiar e comunitária na Atenção Primária à Saúde da criança. Ciênc Saúde Coletiva., 21 (1), 119-127. https://doi.org/10.1590/1413-81232015211.05682014

Reis, R. S., Coimbra, L. C., Silva, A. A. M., Santos, A. M., Britto-Alves, M. T. S. S., Santos, A. M., et al. (2013). Acesso e utilização dos serviços na Estratégia Saúde da Família na perspectiva dos gestores, profissionais e usuários. Ciênc Saúde Coletiva., 18 (11), 3321-3331. https://doi.org/10.1590/S141381232013001100022

Sala, A., Luppi, C. G., Simões, O., \& Marsiglia, R. G. (2011). Integralidade e Atenção Primária à Saúde: avaliação na perspectiva dos usuários de unidades de saúde do município de São Paulo. Saúde Soc., 20 (4), 948-960. https://doi.org/10.1590/S0104-12902011000400012

Santos, N., Veiga, P., \& Andrade, R. (2011). Importância da anamnese e do exame físico para o cuidado do enfermeiro. Rev Bras Enferm., 64 (2), 355 -358. https://doi.org/10.1590/S0034-71672011000200021

Santos, R. P., Horta, P. M., Souza, C. S., Santos, C. A., Oliveira, H. B. S., Almeida, L. M. R., \& Santos, L. C. (2012). Aconselhamento sobre alimentação e atividade física: prática e adesão de usuários da atenção primária. Rev Gaúcha Enferm., 1 (33), 14-21. https://doi.org/10.1590/S1983-14472012000400002

Silva, K. F. (2017). Pcatool-Brasil versão profissionais: avaliação do atributo acesso de primeiro contato na atenção primária à saúde em municípios do interior do Rio Grande do Sul [dissertação]. Santa Maria (RS): Universidade Federal de Santa Maria. https://repositorio.ufsm.br/handle/1/1 1940

Silva, M. F. F., Silva, E. M., Oliveira, S. L. S. S., Abdala, G. A., \& Meira, M. D. D. (2018). Integralidade na atenção primária à saúde. Refacs., 1 (6), $394-400$. http://bvsms.saude.gov.br/bvs/artigos/mundo_saude/integralidade_antecao_saude_olhar_equipe.pdf

Souza, B. R., Tavares, J. B., Girard, C. C. P., \& Ferreira, I. P. (2019). Avaliação da Atenção Primária à Saúde em uma estratégia saúde da família no interior do Pará: Utilização do PCATool-versão Brasil. APS Rev., 1 (2), 112-20. https://apsemrevista.org/aps/article/view/24

Starfield, B. (2002). Atenção primária: equilíbrio entre necessidades de saúde, serviços e tecnologia [Internet]. Brasília: UNESCO; Ministério da Saúde. https://www.nescon.medicina.ufmg.br/biblioteca/imagem/0253.pdf

Sullivan, K. M., Dean, A., \& Soe, M. M. (2009). OpenEpi: A Web-based Epidemiologic and Statistical Calculator for Public Health. Public Health Rep., 124 (3), 471-474. https://doi.org/10.1177/003335490912400320

Vidal, T. B., Tesser, C. D., Harzheim, E., \& Fontanive, P. V. (2018). Avaliação do desempenho da Atenção Primária à Saúde em Florianópolis, Santa Catarina, 2012: estudo transversal de base populacional. Epidemiol Serv Saúde., 27 (4), 504-517. http://dx.doi.org/10.5123/s1679-49742018000400006. 